\title{
CURVATURE APPROXIMATION FROM PARABOLIC SECTORS
}

\author{
X. Gual-ArnaU ${ }^{\bowtie, 1}$, M. V. IbÁÑNEZ ${ }^{2}$ AND J. Monterde 3 \\ ${ }^{1}$ Departament de Matemàtiques, Institute of New Imaging Technologies, Universitat Jaume I. 12071-Castelló, \\ Spain.; ${ }^{2}$ Departament de Matemàtiques, Institute of Mathematics and Applications, Universitat Jaume I. \\ 12071-Castelló, Spain.; ${ }^{3}$ Departament de Geometria i Topologia. Universitat de València. 46100-Burjassot, \\ Spain.
}

e-mail: gual@uji.es,mibanez@uji.es,monterde@uv.es

(Received January 26, 2017; revised May 25, 2017; accepted June 29, 2017)

\begin{abstract}
We propose an invariant three-point curvature approximation for plane curves based on the arc of a parabolic sector, and we analyze how close this approximation is to the true curvature of the curve. We compare our results with those obtained with other invariant three-point curvature approximations. Finally, an application is discussed.
\end{abstract}

Keywords: curvature, digital curve, shape analysis, three-point curvature approximation.

\section{INTRODUCTION}

Curvature is an essential concept in geometry and plays a crucial role in shape description and characterization, being particularly important in problems related to feature extraction, geometry processing and segmentation algorithms. The curvature of a smooth plane curve is defined as the inverse of the radius of its osculating circle at each point. If a parametric representation $\alpha(s)=$ $\{x(s), y(s)\}$ of the curve is available, the expression to compute its signed curvature becomes

$$
\tilde{\kappa}=\frac{x^{\prime} y^{\prime \prime}-y^{\prime} x^{\prime \prime}}{\left(x^{\prime 2}+y^{\prime 2}\right)^{\frac{3}{2}}},
$$

where primes refer to derivatives with respect to the parameter $s$. The curvature is $\kappa=|\tilde{\kappa}|$. As any shape descriptor, curvature is invariant by the action of the group of motions. However a problem of the curvature invariant is that derivatives have to be computed, amplifying the effect of noise. To overcome this limitation, Manay et al. (2004) introduced integral invariant functionals for shapes represented as closed planar contours, as opposed to the traditional differential ones and they proved that such functionals are far less sensitive to noise, while retaining the nice features of differential invariants such as locality; that is, they obtained results which are used to calculate curvature based on integral computing. Pottmann et al. (2009) extended the application of these integral invariant functionals and others defined via distance functions, to surfaces in $\mathbb{R}^{3}$, where, using Taylor expansions, they showed the relation of these integral invariants to differential invariants, as curvature. Other papers where these integral invariant functionals are used are Pottmann et al. (2007) and Lai et al. (2009).

Although curvature estimation from a digital curve would seem rather simple, there don't exist many methods that are easy to implement, fast, and robust to noise and to digitization effects. Four main approaches have been used in the literature to estimate the curvature of a digital curve (Worring and Smeulders, 1992; Hermann and Klette, 2006): orientation based, angle-based, derivative-based and osculating circlebased.

The orientation-based methods estimate the curvature by analyzing the change in slope of the tangent at each point of the curve in a certain direction. The angle-based approach estimates the curvature at each point $P_{i}$ by considering the cosine of the angle formed by the vectors $\overrightarrow{P_{i} P_{i-k}}$ and $\overrightarrow{P_{i} P_{i+k}}$. For the derivative-based approach, the curve is approached by second order curves such as splines to obtain an approximation to the parametric expression of the curve. Finally, in the osculating circle-based approach (Calabi et al., 1998), after smoothing, the boundary is fitted by a circular disc of a certain radius; then, the curvature is computed using the inverse of these radii at each point. To avoid digital effects, in all these curvature estimations, some continuous approximation is attempted when estimating the tangents at the boundary, or when the boundary is approximated to obtain a parametric definition, or when a circle is fitted to a smoothed boundary. This may introduce errors in the boundary or even in the shape.

Since curvature is a second order differential function, the simplest approximation of the curvature of a plane curve $\mathscr{C}$, which is represented by a 
sequence of mesh points $\left\{P_{1}, \cdots, P_{N}\right\}$, not necessarily equally spaced, will require three mesh points (Calabi et al., 1996). Given three non-collinear points, $P_{a}=$ $\left(x_{a}, y_{a}\right), P_{i}=\left(x_{i}, y_{i}\right), P_{b}=\left(x_{b}, y_{b}\right)$, there exists a unique circle passing through them whose radius can be computed by

$$
r_{\text {circle }}=\frac{\left\|P_{b}-P_{i}\right\|\left\|P_{a}-P_{i}\right\|\left\|P_{b}-P_{a}\right\|}{2\left|\left(x_{i}-x_{b}\right)\left(y_{a}-y_{i}\right)+\left(x_{a}-x_{i}\right)\left(y_{b}-y_{i}\right)\right|},
$$

where $\left\|P_{k}-P_{l}\right\|$ is the distance between $P_{k}$ and $P_{l}$. Therefore, the osculating circle-approach to the curvature, $\kappa_{\text {circle, }}$, at $P_{i}$, is given by the inverse of this $r_{\text {circle. }}$. Meanwhile if $\psi$ is the positive angle, defined by the vectors $P_{b}-P_{i}$ and $P_{a}-P_{i}$ the curvature $\left(\kappa_{\text {angle }}\right)$ at $P_{i}$ can also be approached (Belyaev, 1999) as

$$
\kappa_{\text {angle }}\left(P_{i}\right)=\frac{2 \psi}{\left\|P_{b}-P_{i}\right\|+\left\|P_{a}-P_{i}\right\|} .
$$

Given any regular, smooth plane curve $\mathscr{C}$ approximated by a particular sequence of mesh points $\left\{P_{i}\right\}_{i=1, \cdots, N}$, our aim in this paper is to propose a new three-point approximation method of the curvature of $\mathscr{C}$ at $P_{i}$ from the area of a parabolic sector. In order to analyze the goodness of our numerical approach we will consider a Taylor series expansion of the approximated curvature in terms of the true curvature and its derivatives with respect to arc-length, and we will compare it with a similar expansion for the known angle-based and osculating circle-based methods.

So in subsection "Approach" an approach to estimate the curvature based on geometric elements of a parabolic sector is introduced. The goodness of the approach is analyzed in subsection "Goodness of the approach", where a comparison with the performance of other three-point curvature approximation methods is analyzed. Finally, this approach is applied in section "Application: Detection of cells in clusters" to a real application.

\section{A THREE-POINT CURVATURE APPROXIMATION FROM A PARABOLIC SECTOR}

Given $P_{1}, P_{2}, \ldots, P_{N}$ the points defining the mesh of a plane curve $\mathscr{C}$, we will prove in the next lemma that, for any three consecutive points $P_{i-1}, P_{i}$ and $P_{i+1}$, there exists a unique parabola $\mathfrak{P}$ whose tangent line at $z=P_{i}$ is parallel to the segment defined by $P_{i-1}$ and $P_{i+1}$, i.e. such that the unit tangent vector to the parabola at $P_{i}$ is $u_{i}=\frac{P_{i+1}-P_{i-1}}{\left\|P_{i+1}-P_{i-1}\right\|}$.
Lemma 1 Given three non collinear points in $\mathbb{R}^{2}$, $P_{i-1}, P_{i}$ and $P_{i+1}$, there is a unique parabola $\mathfrak{P}$ passing through them and such that the tangent line at $P_{i}$ is parallel to the segment $P_{i-1} P_{i+1}$.

Proof.- Let us choose a coordinate system with $P_{i}$ as origin of coordinates and where the segment $P_{i-1} P_{i+1}$ is parallel to the $x$-axis. Moreover we can suppose that $P_{i-1}$ and $P_{i+1}$ are located above the $x$-axis. Therefore, if we suppose that the coordinates of the points $P_{i-1}$ and $P_{i+1}$ are

$$
P_{i-1}=\left(x_{i-1}, y_{i-1}\right), \quad P_{i+1}=\left(x_{i+1}, y_{i+1}\right),
$$

then $y_{i-1}=y_{i+1}>0$.

The general equation of a parabola passing through $P_{i}=(0,0)$ is

$$
(a x+b y)^{2}+d x+e y=0 .
$$

Moreover, if the tangent line at $P_{i}$ is parallel to the $x$ axis, then $d=0$. Therefore, the equation is reduced to

$$
(a x+b y)^{2}+e y=0 \text {. }
$$

If the parabola passes through $P_{i-1}=\left(x_{i-1}, y_{i-1}\right)$ and $P_{i+1}=\left(x_{i+1}, y_{i-1}\right)$, then

$$
\begin{aligned}
& \left(a x_{i-1}+b y_{i-1}\right)^{2}+e y_{i-1}=0, \\
& \left(a x_{i+1}+b y_{i-1}\right)^{2}+e y_{i-1}=0 .
\end{aligned}
$$

Therefore

$$
a x_{i-1}+b y_{i-1}= \pm\left(a x_{i+1}+b y_{i-1}\right) .
$$

The positive case gives $x_{i-1}=x_{i+1}$ in contradiction with $P_{i-1} \neq P_{i+1}$. The negative case gives the only possible solution. Indeed, from

$$
a x_{i-1}+b y_{i-1}=-a x_{i+1}-b y_{i-1}
$$

we get

$$
2 b y_{i-1}=-a\left(x_{i-1}+x_{i+1}\right),
$$

and

$$
b=-\frac{a\left(x_{i-1}+x_{i+1}\right)}{2 y_{i-1}} .
$$

And, finally, the only possible parabola is determined with

$$
e=-\frac{\left(a x_{i-1}+b y_{i-1}\right)^{2}}{y_{i-1}} .
$$

Additionally, there is always a suitable rotation and translation such that this parabola is transformed in a parabola with equation $y=a x^{2}+b x$, where the corresponding point to $P_{i}$ is $z=P_{0}=(0,0)$, (Fig. 1). As curvature is invariant by the action of the group of motions both parabolas keep the value of the curvature on all the corresponding points. Our aim is to approach the curvature of $\mathscr{C}$ at $P_{i}$ by the curvature of this parabola at $P_{0}$. 


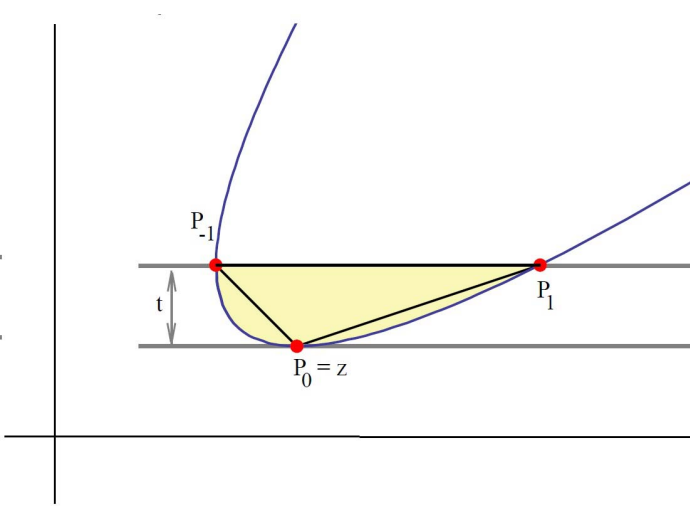

(a)

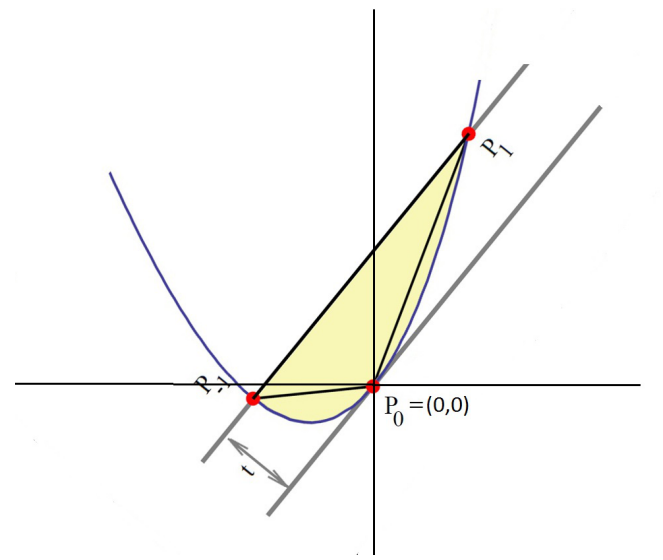

(b)

Fig. 1. The parabola defined by three points that verifies that the tangent in $P_{0}$ is parallel to the segment $\overline{P_{-1} P_{1}}(a)$, and the same parabola rotated and translated $(b)$.

\section{APPROACH}

Theorem 1 Given $P_{0}$ a point on a parabola, let us consider the parabolic sector defined by the parabola, and a line parallel to the tangent line to the parabola at $P_{0}$. Then, the curvature, $\kappa_{\text {parabola }}$, of the parabola at $P_{0}$ can be computed as

$$
\kappa_{\text {parabola }}\left(P_{0}\right)=\frac{32}{9} \frac{t^{3}}{A^{2}(t)},
$$

where $t$ (spacing) is the distance between the two parallel lines and $A(t)$ is the area of the parabolic sector (Fig. 1 a).

Proof.- Due to the invariance of the curvature by rigid motions, let us assume a parabola of equation $y=a x^{2}+b x$, with $a \neq 0$, where $P_{0}=\left(x_{0}, y_{0}\right)=(0,0)$. According to Eq. 1 its curvature at $P_{0}=(0,0)$ is

$$
\kappa_{\text {parabola }}\left(P_{0}\right)=\frac{2 a}{\left(1+b^{2}\right)^{\frac{3}{2}}} .
$$

Let us build parabolic sectors related to $P_{0}$ in the sense that the straight segments defining them are parallel to the tangent line to the parabola at $P_{0}$.

As the tangent line at the point $P_{0}=(0,0)$ is given by the implicit equation

$$
\langle(x, y)-(0,0),(-b, 1)\rangle=0,
$$

parallel lines at a distance $t \in \mathbb{R}^{+}$are given by

$$
\left\langle(x, y)-(0,0), \frac{(-b, 1)}{\sqrt{1+b^{2}}}\right\rangle=t .
$$

After simplification,

$$
y=b x+t \sqrt{1+b^{2}} .
$$

So, the intersection between one of these lines and the parabola fulfills:

$$
b x+t \sqrt{1+b^{2}}=a x^{2}+b x,
$$

which has the solutions

$$
x= \pm \sqrt{\frac{t}{a}\left(1+b^{2}\right)^{\frac{1}{2}}} .
$$

The coordinates of the two points determined on the parabola are

$$
P_{ \pm 1}=\left( \pm \sqrt{\frac{t}{a}\left(1+b^{2}\right)^{\frac{1}{2}}}, \pm b \sqrt{\frac{t}{a}\left(1+b^{2}\right)^{\frac{1}{2}}}+t\left(1+b^{2}\right)^{\frac{1}{2}}\right) .
$$

Therefore, the area of the triangle $T$ defined by $P_{-1}, P_{0}$ and $P_{+1}$ is

$$
A(T)=\frac{1}{2}\left|\left(P_{-1}-P_{0}\right) \wedge\left(P_{+1}-P_{0}\right)\right|=\frac{t^{\frac{3}{2}}}{a^{\frac{1}{2}}}\left(1+b^{2}\right)^{\frac{3}{4}} .
$$

As the area of a parabolic sector $A(t)$ is four-thirds that of its inscribed triangle (Archimedes, 1952),

$$
A(t)=\frac{4}{3} \frac{t^{\frac{3}{2}}}{a^{\frac{1}{2}}}\left(1+b^{2}\right)^{\frac{3}{4}}
$$

According to Eq. $3,\left(1+b^{2}\right)^{\frac{3}{4}}=\sqrt{\frac{2 a}{\kappa_{\text {parabola }}\left(P_{0}\right)}}$, then

$$
A(t)=\frac{4}{3} \frac{t^{\frac{3}{2}}}{a^{\frac{1}{2}}} \sqrt{\frac{2 a}{\kappa_{\text {parabola }}\left(P_{0}\right)}}=\frac{4 \sqrt{2}}{3} \frac{t^{\frac{3}{2}}}{\kappa_{\text {parabola }}\left(P_{0}\right)^{\frac{1}{2}}} .
$$

and Eq. 2 can be easily deduced. 
Corollary 1 Given three non-collinear points $P_{-1}, P_{0}$ and $P_{+1}$, the curvature of the unique parabola $\mathfrak{P}$ through $P_{-1}, P_{0}$ and $P_{+1}$, whose unit tangent vector at $P_{0}$ is $u_{0}=\frac{P_{+1}-P_{-1}}{\left\|P_{+1}-P_{-1}\right\|}$, is given by:

$$
\kappa_{\text {parabola }}\left(P_{0}\right)=\frac{32}{9} \frac{t^{3}}{A^{2}(t)}=\frac{2 t^{3}}{A(T)^{2}}=\frac{8 t}{h^{2}}
$$

where $A(T)=\frac{t h}{2}$ is the area of the triangle defined by $P_{-1}, P_{0}$ and $P_{+1}, h=\left\|P_{+1}-P_{-1}\right\|$ and $t=$ $\operatorname{dist}\left(P_{0}, \overline{P_{-1} P_{+1}}\right), \overline{P_{-1} P_{+1}}$ denotes the line joining $P_{-1}$ and $P_{+1}$.

Let $\mathfrak{P}_{2}$ be another parabola through $P_{0}$ whose tangent line $L$ at $P_{0}$ is given by the unit vector $u_{0}=$ $\frac{P_{+1}-P_{-1}}{\left\|P_{+1}-P_{-1}\right\|}$; and let $P_{-2}, P_{+2}$ be the intersection points between $\mathfrak{P}_{2}$ and the parallel line to $L$ at a distance $t$; then, from Theorem 1, if the area $A\left(T_{2}\right)$ of the triangle defined by $P_{-2}, P_{0}, P_{+2}$ coincides with $A(T)$; that is, $\left\|P_{+1}-P_{-1}\right\|=\left\|P_{+2}-P_{-2}\right\|=h$; then the curvature of the parabolas $\mathfrak{P}$ and $\mathfrak{P}_{2}$ at $P_{0}$ coincide; and its value is given by the Eq. 4. In Fig. 2 we have three different parabolas with $\left\|P_{+1}-P_{-1}\right\|=\left\|P_{+2}-P_{-2}\right\|=\| P_{+3}-$ $P_{-3} \|=h$, and therefore with the same curvature at $P_{0}$.

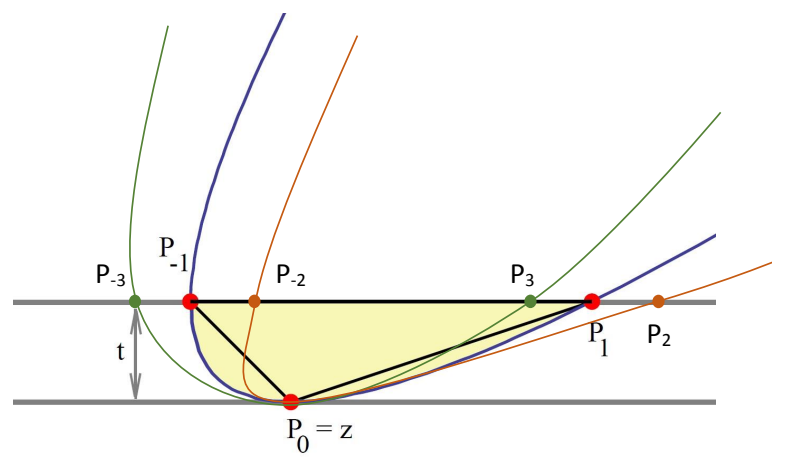

Fig. 2. Parabolic sectors with the same curvature at $P_{0}$.

Eq. 4 allows to relate our curvature approximation with the concept of curvature scale introduced in Rueda et al. (2008; 2010), taking then into account the digital effects of noise and considering scale in curvature approximation.

\section{GOODNESS OF THE APPROACH}

To analyze the goodness of this approach, let $\mathscr{C}$ be a known smooth plane curve, and lets approach the curvature $\kappa$ of $\mathscr{C}$ at a point $P_{0} \in \mathscr{C}$, from the curvature $\kappa_{\text {parabola }}$ of a parabola defined from $\mathscr{C}, P_{0}$, a spacing parameter $t$, and the tangent line to $\mathscr{C}$ at $P_{0}$.

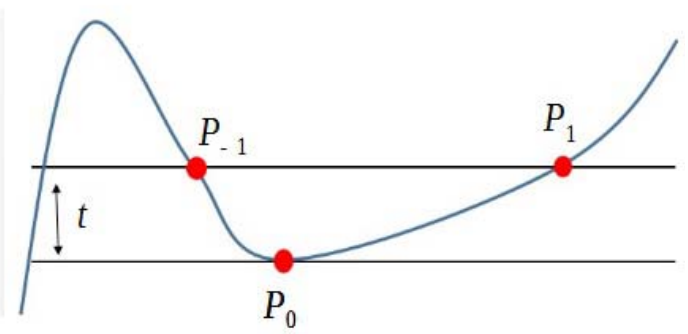

Fig. 3. Smooth plane curve $\mathscr{C}$.

Theorem 2 Let $P_{0}$ be a point on a smooth plane curve $\mathscr{C}$, and let $\kappa$ denotes the curvature of the curve $\mathscr{C}$ at $P_{0}$. For any small enough given spacing $t$, let us consider the two points $P_{-1}$ and $P_{1}$, intersecting the curve with the line parallel, at a distance $t$, to the tangent line of $\mathscr{C}$ at $P_{0}$ (Fig. 3).

Let $\kappa_{\text {parabola }}$ denote the curvature at $P_{0}$ of the parabola passing through $P_{-1}, P_{0}$ and $P_{1}$ and having the same tangent line at $P_{0}$ as the curve $\mathscr{C}$ (Fig. la). Then:

$$
\kappa_{\text {parabola }}=\kappa+\left(\frac{9 \kappa^{4}-5 \kappa^{\prime 2}+3 \kappa \kappa^{\prime \prime}}{18 \kappa^{2}}\right) t+O\left(t^{2}\right) .
$$

Proof.- Given $P_{0}, P_{1}$ and $P_{-1} \in \mathscr{C}$, the parabola passing through them and having the tangent line at $P_{0}$ parallel to $\overline{P_{1} P_{-1}}$ can be built. Then, applying Eq. 3, the curvature of this parabola at $P_{0}$ is:

$$
\kappa_{\text {parabola }}=\frac{32}{9} \frac{t^{3}}{A(t)^{2}} .
$$

If $\alpha: I \rightarrow \mathbb{R}^{2}$ denotes the arc length parametrization of the curve $\mathscr{C}$,

$$
\begin{aligned}
\alpha(s) & =\alpha(0)+s \overrightarrow{\mathbf{t}}(0)+\frac{s^{2}}{2} \kappa(0) \overrightarrow{\mathbf{n}}(0) \\
& +\frac{s^{3}}{6}\left(\kappa^{\prime}(0) \overrightarrow{\mathbf{n}}(0)-\kappa^{2}(0) \overrightarrow{\mathbf{t}}(0)\right) \\
& +\frac{s^{4}}{24}\left(-3 \kappa(0) \kappa^{\prime}(0) \overrightarrow{\mathbf{t}}(0)+\left(\kappa^{\prime \prime}(0)-\kappa^{3}(0)\right) \overrightarrow{\mathbf{n}}(0)\right) \\
& +O\left(s^{5}\right)
\end{aligned}
$$

So:

$$
\begin{array}{r}
\alpha(s)=\alpha(0)+\left(s-\kappa^{2}(0) \frac{s^{3}}{6}-\kappa(0) \kappa^{\prime}(0) \frac{s^{4}}{8}\right) \overrightarrow{\mathbf{t}}(0)+ \\
+\left(\frac{s^{2}}{2} \kappa(0)+\frac{s^{3}}{6} \kappa^{\prime}(0)+\left(\kappa^{\prime \prime}(0)-\kappa^{3}(0)\right) \frac{s^{4}}{24}\right) \overrightarrow{\mathbf{n}}(0)+ \\
+O\left(s^{5}\right),
\end{array}
$$

with $\alpha^{\prime}(0)=\overrightarrow{\mathbf{t}}(0)$ the unit tangent vector at $P_{0}$ and $\alpha^{\prime \prime}(0)=\kappa \overrightarrow{\mathbf{n}}(0)$ with $\overrightarrow{\mathbf{n}}(0)$ the unit normal vector at $P_{0}$ and $\kappa$ the curvature without sign. 
It is necessary to consider until the fourth term of the expansion in order to get the right coefficient of $t$ in Eq. 5.

Since $P_{-1}, P_{0}$ and $P_{1}$ are points of $\alpha$, we suppose that the point $P_{0}$ is $\alpha(0)$, the point $P_{1}$ is of the kind $\alpha\left(s_{1}\right)$ and the point $P_{-1}$ is $\alpha\left(-s_{-1}\right)$ with $s_{-1}>0$. Moreover, since the tangent vector at $\alpha(0)$ is parallel to the line joining $P_{-1}$ and $P_{1}$, the normal vector at $\alpha(0)$ is perpendicular to this line. Thus the normal component of the vectors $\overrightarrow{P_{0} P_{1}} \overrightarrow{P_{0} P_{-1}}$ should be $t$, and we can approximate this distance $t$ between the parallel lines in two different ways:

$$
\begin{aligned}
& t=\frac{s_{1}^{2}}{2} \kappa+\frac{s_{1}^{3}}{6} \kappa^{\prime}+\left(\kappa^{\prime \prime}-\kappa^{3}\right) \frac{s_{1}^{4}}{24}+O\left(s_{1}^{5}\right), \quad \text { or } \\
& t=\frac{s_{-1}^{2}}{2} \kappa-\frac{s_{-1}^{3}}{6} \kappa^{\prime}+\left(\kappa^{\prime \prime}-\kappa^{3}\right) \frac{s_{-1}^{4}}{24}+O\left(s_{-1}^{5}\right) .
\end{aligned}
$$

Inverting both series we get

$$
\begin{gathered}
s_{1}=\sqrt{\frac{2 t}{\kappa}}-\frac{\kappa^{\prime}}{3 \kappa^{2}} t+\frac{3 \kappa^{4}+5 \kappa^{\prime 2}-3 \kappa \kappa^{\prime \prime}}{18 \sqrt{2} \kappa^{7 / 2}} t^{3 / 2}+O\left(t^{2}\right), \\
s_{-1}=\sqrt{\frac{2 t}{\kappa}}+\frac{\kappa^{\prime}}{3 \kappa^{2}} t+\frac{3 \kappa^{4}+5 \kappa^{\prime 2}-3 \kappa \kappa^{\prime \prime}}{18 \sqrt{2} \kappa^{7 / 2}} t^{3 / 2}+O\left(t^{2}\right) .
\end{gathered}
$$

Next, since the tangential coefficient of $P_{0} P_{1}$ is of the kind $s_{1}-\kappa^{2} s_{1}^{3} / 6+O\left(s_{1}^{4}\right)$ (see Eq. 6), and analogously for $P_{0} P_{-1}$, the basis of the triangle $P_{-1} P_{0} P_{1}$ is, up to fourth order, the same as $\left(s_{1}+s_{-1}\right)-$ $\left(\kappa^{2} / 6\right)\left(s_{1}^{3}+s_{-1}^{3}\right)$, whereas its height is $t$; therefore, the area $A(T)$ of the triangle $P_{-1} P_{0} P_{1}$ is:

$$
\begin{aligned}
A(T) & =\frac{t\left(\left(s_{1}+s_{-1}\right)-\frac{\kappa^{2}}{6}\left(s_{1}^{3}+s_{-1}^{3}\right)\right)}{2}= \\
& =\sqrt{\frac{2}{\kappa}} t^{3 / 2}+\frac{5 \kappa^{\prime 2}-3 \kappa \kappa^{\prime \prime}-9 \kappa^{4}}{18 \sqrt{2} \kappa^{7 / 2}} t^{5 / 2}+O\left(t^{3}\right) .
\end{aligned}
$$

Applying again that the area of the parabolic sector $A(t)$ is four-thirds that of the inscribed triangle $A(T)$;

$$
\begin{gathered}
A(t)^{2}=\left(\frac{4}{3} A(T)\right)^{2} \\
=\frac{16}{9}\left[\sqrt{\frac{2}{\kappa}} t^{3 / 2}+\frac{5 \kappa^{\prime 2}-3 \kappa \kappa^{\prime \prime}-9 \kappa^{4}}{18 \sqrt{2} \kappa^{7 / 2}} t^{5 / 2}+O\left(t^{3}\right)\right]^{2} \\
=\frac{16}{9}\left[\frac{2}{\kappa} t^{3}+2 \sqrt{\left.\frac{2}{\kappa}\left(\frac{5 \kappa^{\prime 2}-3 \kappa \kappa^{\prime \prime}-9 \kappa^{4}}{18 \sqrt{2} \kappa^{7 / 2}}\right) t^{4}+O\left(t^{5}\right)\right]}\right. \\
=\frac{32}{9}\left[\frac{t^{3}}{\kappa}+\left(\frac{5 \kappa^{\prime 2}-3 \kappa \kappa^{\prime \prime}-9 \kappa^{4}}{18 \kappa^{4}}\right) t^{4}+O\left(t^{5}\right)\right],
\end{gathered}
$$

and

$$
\begin{aligned}
& \kappa_{\text {parabola }}=\frac{32}{9} \frac{t^{3}}{A(t)^{2}}= \\
& =\kappa+\left(\frac{9 \kappa^{4}-5 \kappa^{\prime 2}+3 \kappa \kappa^{\prime \prime}}{18 \kappa^{2}}\right) t+O\left(t^{2}\right)
\end{aligned}
$$

The coefficient $\frac{9 \kappa^{4}-5 \kappa^{12}+3 \kappa \kappa^{\prime \prime}}{18 \kappa^{2}}$ is a fourth order differential invariant of plane curves in the Euclidean space. If the curve is parametrized as $\alpha(u)=(u, f(u))$, then

$$
\frac{9 \kappa^{4}-5 \kappa^{\prime 2}+3 \kappa \kappa^{\prime \prime}}{18 \kappa^{2}}=\frac{3 f^{\prime \prime} f^{(i v)}-5\left(f^{\prime \prime \prime}\right)^{2}}{18\left(1+\left(f^{\prime}\right)^{2}\right)\left(f^{\prime \prime}\right)^{2}} .
$$

Now, it is easy to check that when the curve is the parabola $\left(u, u^{2}\right)$ this coefficient of $t$ vanishes.

\section{Comparison with other three point curvature approximation methods}

As stated below, the three-point curvature estimation methods more widely used in the literature are the osculating circle approach and the approach based on the rate of change of the angle between the tangent and the positive direction of the $x$-axis when we proceed along the curve.

In Calabi et al. (1998) and Belyaev (1999), it is shown that Taylor expansions of $\kappa_{\text {circle }}$ and $\kappa_{\text {angle }}$ with respect to the lengths $\Delta_{i+1}=\left\|P_{i+1}-P_{i}\right\|$ and $\Delta_{i-1}=$ $\left\|P_{i-1}-P_{i}\right\|$, are linear and start from

$$
\begin{aligned}
\kappa_{\text {circle }}= & \kappa+\frac{\Delta_{i+1}-\Delta_{i-1}}{3} \kappa^{\prime}+ \\
& +\frac{1}{12} \frac{\left(\Delta_{i+1}^{3}+\Delta_{i-1}^{3}\right)}{\left(\Delta_{i+1}+\Delta_{i-1}\right)} \kappa^{\prime \prime}+\ldots \\
\kappa_{\text {angle }}= & \kappa+\frac{\Delta_{i+1}-\Delta_{i-1}}{3} \kappa^{\prime}+ \\
& +\frac{1}{12} \frac{\left(\Delta_{i+1}^{3}+\Delta_{i-1}^{3}\right)}{\left(\Delta_{i+1}+\Delta_{i-1}\right)}\left(\kappa^{\prime \prime}+\frac{\kappa^{3}}{2}\right)+\ldots
\end{aligned}
$$

From Eq. 5, $\kappa_{\text {parabola }}=\kappa+\left(\frac{9 \kappa^{4}-5 \kappa^{12}+3 \kappa \kappa^{\prime \prime}}{18 \kappa^{2}}\right) t+$ $O\left(t^{2}\right)$, with $t$ as in Eq. 7. From this expression, and the relations between $\left\|P_{i+1}-P_{i}\right\|$ and $s_{1}$, and $\left\|P_{i-1}-P_{i}\right\|$ and $s_{-1}$, via Taylor series (see, for instance, Belyaev, 1999), the Taylor expansion of our approximation is quadratic and starts from

$$
\begin{aligned}
\kappa_{\text {parabola }}= & \kappa+\frac{\kappa^{2}}{4}\left(\frac{\kappa}{2}-\frac{5 \kappa^{\prime 2}}{18 \kappa^{3}}+\frac{\kappa^{\prime \prime}}{6 \kappa^{2}}\right)\left(\left\|P_{i+1}-P_{i}\right\|^{2}+\right. \\
& \left.+\left\|P_{i-1}-P_{i}\right\|^{2}\right)+\ldots
\end{aligned}
$$

Therefore, supposing that $\kappa, \kappa^{\prime}, \kappa^{\prime \prime}$ are finite, $\kappa$ does not vanish and $\left\|P_{i+1}-P_{i}\right\| \neq\left\|P_{i-1}-P_{i}\right\|$, we 
can always choose the points $P_{i-1}, P_{i}$ and $P_{i+1}$ close enough to ensure that our approximation is better than the osculating circle-based and the orientation-based approaches (Eq. 8).

However, if $\left\|P_{i+1}-P_{i}\right\|=\left\|P_{i-1}-P_{i}\right\|$ the Taylor expansions of $\kappa_{\text {circle }}$ and $\kappa_{\text {angle }}$ (Calabi et al., 1998; Belyaev, 1999) start from

$$
\kappa+\frac{\left\|P_{i+1}-P_{i}\right\|^{2}}{12}\left(\kappa^{\prime \prime}+C \kappa^{3}\right)+\ldots
$$

where $C$ is constant, quite similar to the Taylor expansion of our approximation that starts from

$$
\kappa+\frac{\left\|P_{i+1}-P_{i}\right\|^{2}}{12}\left(\kappa^{\prime \prime}+3 \kappa^{3}-\frac{5 \kappa^{\prime 2}}{3 \kappa}\right)+\ldots
$$

\section{Example}

As an example, let us consider the curve, defined in polar coordinates by

$$
\alpha(s)=\rho(s)(\cos s, \sin s)
$$

where

$$
\begin{aligned}
\rho(s)= & 5+\cos (s)-\frac{1}{2} \cos (2 s)+\sin (2 s)+ \\
& +\cos (3 s)-\frac{2}{5} \sin (3 s)-\frac{3}{4} \cos (4 s)
\end{aligned}
$$

and its discretization in $N$ non-equally spaced points defined by:

$$
P_{i}=\alpha\left(-\pi+i \frac{2 \pi}{N}\right), \quad i \in\{1, \ldots, N\},
$$

and in $N$ equally spaced points in terms of arc length along the curve. Both discretizations are plotted in Fig. 4.

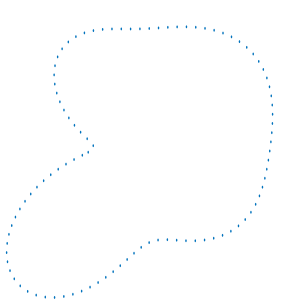

(a) (b)

Fig. 4. Discretized curve considered in the example $(N=100)$. a) Equally spaced points. b) Non equally spaced points.

Three different approaches to estimate the curvature $\left(\kappa_{\text {circle }}, \kappa_{\text {parabola }}\right.$ and $\left.\kappa_{\text {angle }}\right)$ are computed at each $P_{i}$ with $i \in\{1, \ldots, N\}$.
As the calculus of $\kappa_{\text {parabola }}$ (Eq. 4) depends on a parameter $t$, different $t$-values have been chosen to compute the three estimates. All them guarantee that for each $P_{i}$ there exist other two points $P_{i-k_{i}}$ and $P_{i+k_{i}}$ in the discretization, such that if $r_{k_{i}}$ is the line defined by $\overrightarrow{P_{i+k_{i}} P_{i-k_{i}}}$, then $\operatorname{distance}\left(P_{i}, r_{k_{i}}\right) \leq t$. So, for each $P_{i}$, we will not define the parabola/circle/angle with the points $P_{i-1}, P_{i}$ and $P_{i+1}$, but with $P_{i-k_{i}}, P_{i}$ and $P_{i+k_{i}}$ such that $\operatorname{distance}\left(P_{i}, r_{k_{i}}\right) \leq t$ and distance $\left(P_{i}, r_{k_{i}+1}\right)>t$.

Fig. 5 shows how the curvature at different points is approached by the area of different parabolic sectors.

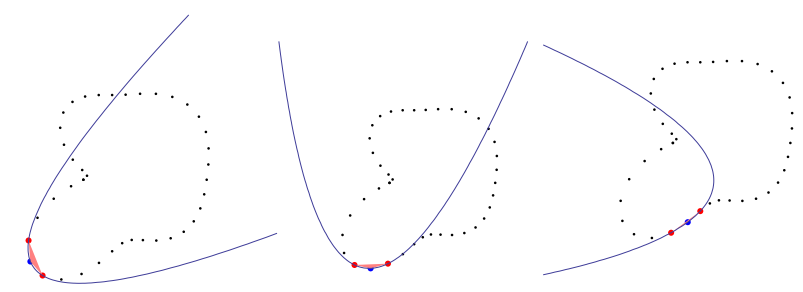

Fig. 5. Three consecutive instances of the computation of the curvature from a discretisation of the curve $\alpha_{1}$.

As the exact curvature at each point is known, the real value can be compared with the obtained with the different approaches. Table 1 shows the mean squared error of the estimates obtained for the curvatures by using the approximations $\kappa_{\text {circle }}, \kappa_{\text {parabola }}$ and $\kappa_{\text {angle }}$ with the different $t$-values.

Table 1. Mean squared error of the estimates obtained for the curvatures by using the approximations $\kappa_{\text {circle, }}$ $\kappa_{\text {parabola }}$ and $\kappa_{\text {angle }}$ with the different $t$-values.

\begin{tabular}{cccc}
\hline \multicolumn{4}{c}{100 equally spaced points } \\
\hline$t$ & $\kappa_{\text {parabola }}$ & $\kappa_{\text {circle }}$ & $\kappa_{\text {angle }}$ \\
\hline 0.1 & & & \\
0.2 & & & \\
0.3 & 0.0278 & 0.0270 & 0.0219 \\
0.4 & 0.0776 & 0.0486 & 0.0470 \\
0.6 & 0.0838 & 0.1105 & 0.1047 \\
0.8 & 0.1085 & 0.1472 & 0.1423 \\
1.0 & 0.1175 & 0.1519 & 0.1470 \\
\hline \multicolumn{4}{c}{100 non-equally spaced points } \\
\hline$t$ & $\kappa_{\text {parabola }}$ & $\kappa_{\text {circle }}$ & $\kappa_{\text {angle }}$ \\
\hline 0.1 & 0.0044 & 0.0426 & 0.0340 \\
0.2 & 0.0472 & 0.2510 & 0.2136 \\
0.3 & 0.0493 & 0.2669 & 0.2225 \\
0.4 & 0.1573 & 0.4746 & 0.4252 \\
0.6 & 0.1685 & 0.5035 & 0.4525 \\
0.8 & 0.3244 & 0.6563 & 0.6117 \\
1.0 & 0.4838 & 0.7673 & 0.7313 \\
\hline
\end{tabular}


As it can be seen, when a discretization of the curve with non equally spaced points is considered and the points are closed enough, the quadratic error of the approximated curvature computed from a parabola is always lower than the quadratic error of the approximated curvature computed from the other methods.

Now, we will consider this same example to illustrate that curvature estimation from Eq. 1, using approximations of the first and second derivatives of $x$ and $y$ (see Sethian, 1996), is less robust to noise than the curvature estimated from a parabolic sector (Eq. 4). In Fig. 6a we consider the same curve as in Fig. 4a and, in Fig. 6b, we consider the same curve corrupted by noise. As it can be observed in Fig. 6c both curvature approximations are good; in fact the mean squared error (MSE) of the curvature estimation using approximations of the derivatives of $x$ and $y$ is 0.0247. However, when a noise effect is introduced, the estimation of the curvature is less robust when derivatives approximation is used. In this case the MSE of the curvature estimation from Eq. 1 is 4.8709 and the MSE of the curvature estimation from Eq. 4 is 0.0560 (for $t=0.5$ ).

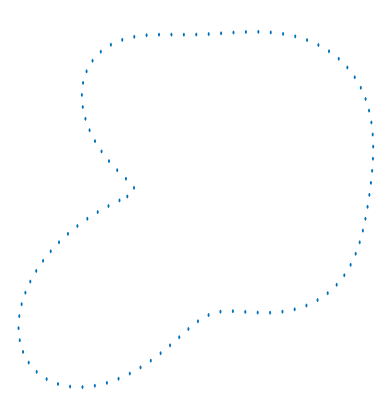

(a)

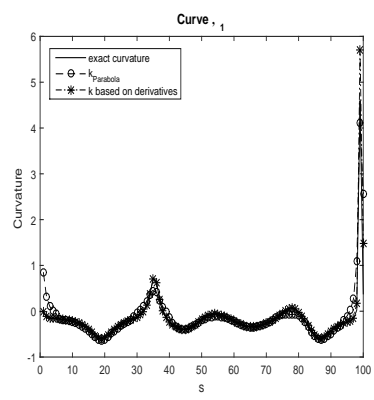

(c)

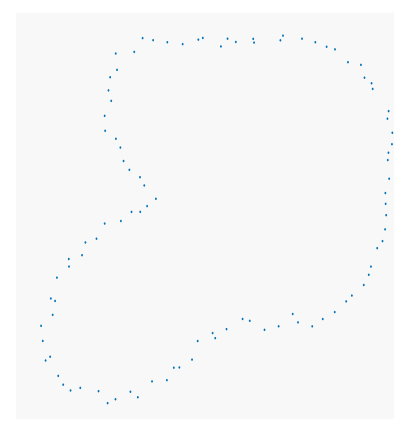

(b)

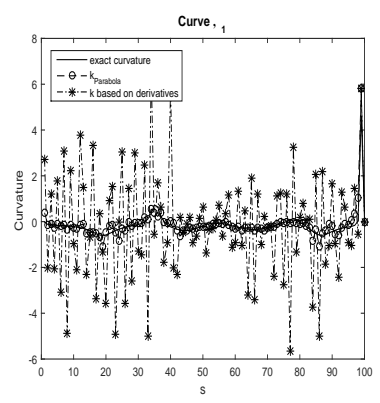

(d)
Fig. 6. (a) Curve defined by Eq. 10 discretized in 100 equally spaced points. (b) Curve defined by Eq. 10 with noise effect introduced. (c) Curvature approximation, original curve. (d) Curvature approximation, curve corrupted by noise.

\section{APPLICATION: DETECTION OF CELLS IN CLUSTERS}

The study of cell morphology is an important aspect of the diagnosis of different diseases, such as sickle cell disease, where red blood cells shape become deformed by the disease Gual-Arnau et al. (2015).

Numerous efforts have recently been made to develop microscopic techniques that allow to visualize real-time cellular images with a high degree of accuracy. In the case of the sickle cell disease, these images must allow to quantify the number of deformed cells and so gauge the severity of the illness. Nowadays, due to the circular or elongated shape of the erythrocyte depending on their status (normal or deformed), ellipse adjustment methods are widely applied to classify the cells. However, due to cells overlapping and clump formation, it is not possible to carry out these tasks in a trivial way. Another method for determining points of interest by considering the changes in curvature in the contour that represents the cells is usually applied. Curvature can also be used to detect in most cases overlapping cells. Points with high negative curvature would signal these intersection points between different cells, and points with high positive curvature would detect the extremes of the elongated cells.

From Eq. 2, the approximation to the signed curvature $\tilde{\kappa}$ at a point $P_{i}$ of $\mathscr{C}$, given three consecutive points $P_{i-1}, P_{i}$ and $P_{i+1}$, is given by

$$
\begin{aligned}
\tilde{\kappa} & \approx \operatorname{sign}\left(\operatorname{Det}\left(\overrightarrow{P_{i-1} P_{i}}, \overrightarrow{P_{i} P_{i+1}}\right)\right) \frac{32}{9} \frac{t^{3}}{A^{2}(t)}= \\
& =\operatorname{sign}\left(\operatorname{Det}\left(\overrightarrow{P_{i-1} P_{i}}, \overrightarrow{P_{i} P_{i+1}}\right)\right) \frac{2 t^{3}}{A^{2}(T)} .
\end{aligned}
$$

Then, when the curve $\mathscr{C}$ is convex at $P_{i}, \tilde{\kappa}>0$ and if $\mathscr{C}$ is concave at $P_{i}, \tilde{\kappa}<0$.

As an illustration, Figs. 7a, d and g show three examples of images with overlapping erythrocytes, obtained from patients with Sickle cell disease. As a first step, several pre-processing algorithms have been applied to obtain noise-free segmented images (Figs. 7b, e and h), and their contours (Figs. 7c, f and i; Serra, 1982). All these steps have been done by using the software Matlab (The MathWorks, 2015). 


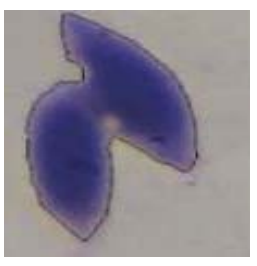

(a)

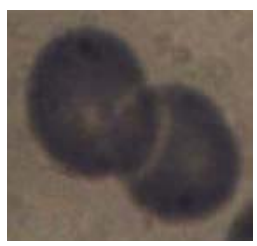

(d)

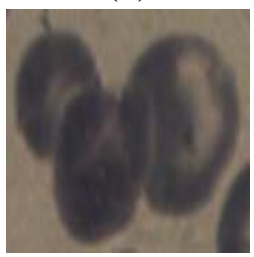

(g)

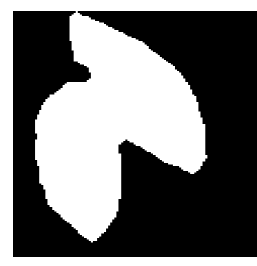

(b)

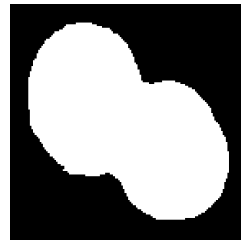

(e)

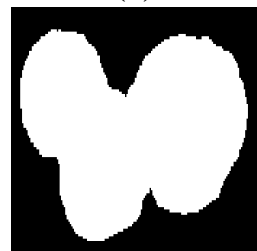

(h)

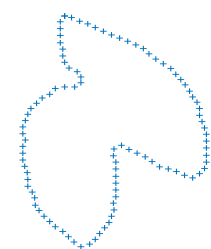

(c)

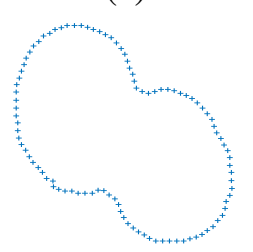

(f)

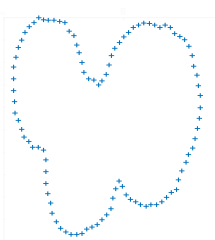

(i)
Fig. 7. (a), (d) and (g) show the original images. (b), $(e)$ and $(h)$ the binary segmented images, and $(c),(f)$ and $(i)$ the discretized contours.

Curvature at each point has been estimated by the three point parabolic approach and by the circle approach. Figs. 8 a), c) and e) show the curvature value at each point. Points with minima curvature are highlighted on the respective contours in figs $8 \mathrm{~b}$ ), d) and f). As it can be observed in these figures, with the parabolic approach it is easier to detect the points with extreme curvature.

\section{ACKNOWLEDGEMENTS}

Work supported by the PROMETEOII/2014/062 project and the Spanish Ministry of Science and Innovation Project DPI2013-47279-C2-1-R.

\section{REFERENCES}

Archimedes (1952). Quadrature of the parabola, (translated by Sir Thomas L. Heath). In: Hutchins RM, ed., Great Books of the Western World, vol. 11. Encyclopedia Britannica, Inc., 527-37.

Belyaev A (1999). A note on invariant three-point curvature approximations. Tech. Rep. 1111:157-64. Kyoto University.

Calabi E, Olver P, Shakiban C, Tannenbaum A, Haker S (1998). Differential and numerically invariant signature curves applied to object recognition. Int $\mathrm{J}$ Comput Vision 26:107-35.

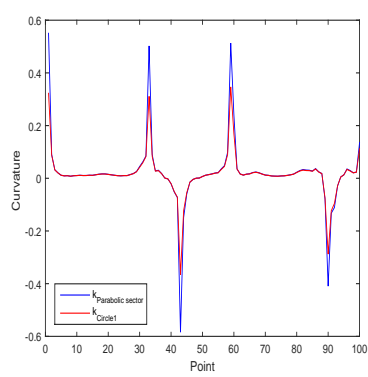

(a)

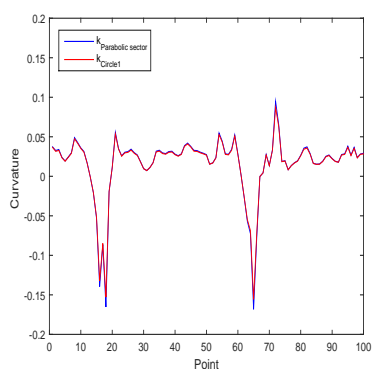

(c)

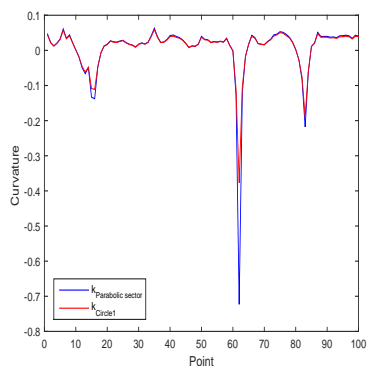

(e) (b)
Fig. 8. (a), (c) and (e) show the three-point curvature approximation from parabolic sectors, jointly with the $\kappa_{\text {circle }}$ approach. $(b),(d)$ and $(f)$ show the points of maximum curvature, which are plotted in green, and the minimum curvature points in red.

Calabi E, Olver PJ, Tannenbaum A (1996). Affine geometry, curve flows, and invariant numerical approximations. Adv Math 124:154-96.

Gual-Arnau X, Herold-García S, Simó A (2015). Erythrocyte shape classification using integralgeometry-based methods. Med Biol Eng Comput 53:623-33.

Hermann S, Klette R (2006). A comparative study on 2d curvature estimators. Tech. rep., CITR, The University of Auckland, New Zealand.

Lai Y, Hu S, Fang T (2009). Robust principal curvatures using feature adapted integral invariants. In: Proc 2009 
SIAM/ACM J Conf Geom Phys Model. 325-330.

Manay S, Hong B, Yezzi A, Soatto S (2004). Integral invariant signatures. In: Pajdla T, Matas J, eds., Computer Vision - ECCV 2004. Lect Not Comput Sci 3024:87-99.

The MathWorks (2015). MATLAB, version R2015b. Natick, Massachusetts: The MathWorks Inc.

Pottmann H, Wallner J, Huang Q, Yang Y (2009). Integral invariants for robust geometry processing. Comput Aided Geom D 26:37-60.

Pottmann H, Wallner J, Yang Y, Lai Y, Hu S (2007). Principal curvatures from the integral invariant viewpoint. Comput Aided Geom D 24:428-42.
Rueda S, Udupa J, Bai L (2008). Local curvature scale: a new concept of shape description. In: Proc Med Imaging 2008: Image Processing. Proc SPIE 6914:69144Q

Rueda S, Udupa J, Bai L (2010). Shape modeling via local curvature scale. Pattern Recogn Lett 31:324-36.

Serra J (1982). Image analysis and mathematical morphology. London: Academic Press.

Sethian JA (1996). Level set methods: Evolving interfaces in geometry, fluid mechanics, computer vision, and materials sciences. Cambridge: Cambridge University Press.

Worring M, Smeulders A (1992). The accuracy and precision of curvature estimation methods. In: Proc 11 IAPR Int Conf Pattern Recogn C:139-42. 